\title{
Factores de riesgo cardiovascular en escolares entre 7 y 14 años en Cartagena, Colombia, 2009
}

\author{
Cardiovascular risk factors among 7- and 14-year old schoolchildren \\ in Cartagena, Colombia, 2009
}

Alicia N. Alayón, Raimundo Castro-Orozco, Luis Gaviria-Esquivia, Milena Fernández-Franco y Lourdes Benítez-Peña

\begin{abstract}
Universidad de San Buenaventura, Seccional Cartagena. Colombia. alinorala@gmail.com, raimundo_castro_orozco@hotmail.com,luisg_25@hotmail.com,kawaii_io@hotmail.com, lbenitez@usbctg.edu.co

Recibido 15 Junio 2010/Enviado para Modificación 29 Marzo 2011/Aceptado 7 Abril 2011

\section{RESUMEN}

Objetivo Identificar antecedentes familiares, dislipidemias, hiperglucemia, escasa actividad física y sobrepeso u obesidad como factores de riesgo cardiovascular en niños entre 7 y 14 años de Cartagena, 2009.

Materiales y Métodos Se realizó un estudio descriptivo en 173 niños de Cartagena, Colombia. Se determinaron los parámetros bioquímicos en suero mediante técnicas espectrofotométricas. Los hábitos fueron indagados mediante entrevista y las alteraciones de peso fueron establecidas utilizando el índice de masa corporal.

Resultados La muestra quedó conformada por 87 niñas y 86 niños con edad promedio de 9,9 años (IC95 \% 9,6-10,3). El 75,1\% (IC95 \% 68,7-81.5) presentaron antecedentes familiares; $2,3 \%$ (IC95 \% 0,1-4,5) tuvieron sobrepeso y 1,7 \% (IC95 \% 0,0-3,6) obesidad. Más de la mitad de los niños presentaron niveles elevados de colesterol total (53,2\%; IC95 \% 45,8-60,6) y LDL (57,2 \%; IC95 \% 49,8-64,6). El colesterol no HDL se halló incrementado en el 46,8 \% (IC95 \% 39,4-54,2). Las niñas presentaron mayor concentración de triglicéridos (94,1 mg/dL; IC95 \% 93,0-95,2 mg/dL) que los niños $(81,7 \mathrm{mg} / \mathrm{dL}$; IC95 \% 80,6-82,8 mg/dL) con diferencia significativa $(\mathrm{p}=0,005)$ y también presentaron con mayor frecuencia baja actividad física (niñas: 83,8 \%; IC95 \% 73,594,5 \%; varones: 44,2 \%; IC95 \% 45,4-66,6; p=0,0001).

Conclusión Los factores de riesgo cardiovascular presentes en esta población, inclusive en ausencia de obesidad, muestran la necesidad de implementar programas que los identifiquen e intervengan de manera oportuna para disminuir su impacto en la calidad de vida futura.

Palabras Clave: Factores de riesgo, enfermedades cardiovasculares, promoción de la salud, prevención y control (fuente: DeCS, BIREME). 


\section{ABSTRACT}

Objective Identifying family history, dyslipidaemia, hyperglycaemia, low physical activity and being overweight or suffering from obesity as cardiovascular risk factors in children aged 7 to 14 years in Cartagena, 2009.

Materials and Methods A descriptive study of 173 children from Cartagena, Colombia, was designed. Biochemical serum parameters were determined by spectrophotometric methods. Habits were investigated through interviews and altered body weight was established using the body mass index (BMI).

Results The sample consisted of 87 girls and 86 boys, and mean age was 9.9 years (9.6-10.3, $95 \% \mathrm{Cl}) .75 .1 \%(68.7-81.5,95 \% \mathrm{Cl})$ had a family history of being overweight;2.3\% $(0.1-4.5,95 \% \mathrm{Cl})$ were overweight and $1.7 \%(0.0-3.695 \% \mathrm{Cl})$ were obese. More than half of the children had hightotal cholesterol levels $(53.2 \%$; 45.8$60.695 \% \mathrm{Cl})$ and LDL (57.2 \%; 49.8-64.6 $95 \% \mathrm{Cl})$. Non-HDL cholesterol was found to be increased by $46.8 \%(39.4-54.295 \% \mathrm{Cl})$. There was a significant difference between genders for triglyceridemia (females: $94.1 \mathrm{mg} / \mathrm{dL} ; 87.0-101.2 \mathrm{mg} / \mathrm{dL} 95 \% \mathrm{Cl}$; males: $81.7 \mathrm{mg} / \mathrm{dL} ; 75.0-88.5 \mathrm{mg} / \mathrm{dL} 95 \% \mathrm{Cl}$; $\mathrm{p}=0.005$ ) and low physical activity (females: 83.8 \%; 73.5-94.5 \% $95 \%$ Cl; males: $44.2 \%$; 45.4-66.6 \% $95 \%$ Cl; p=0,0001). Conclusions Cardiovascular risk factors in these schoolchildren, even in those who were not obese, justify the need for the early detection of these factors as well as their strict controlto reduce their impact on people's future quality of life.

Key Words: Risk factor, cardiovascular disease, health promotion, prevention and control (source: MeSH, NLM).

$\mathrm{S}$ i bien en muchas ocasiones el desenlace de la enfermedad cardiovascular se produce en la vida adulta, sus inicios suceden en edades tempranas. De hecho, se ha reportado presencia de estrías grasas desde la niñez, las cuales evolucionan a placas fibrosas durante la adolescencia y progresan a medida que aumenta la edad (1). La presencia sostenida de factores de riesgo como dislipidemias, obesidad, falta de actividad física e inadecuada alimentación desde la niñez, facilita el desarrollo de enfermedad cardiovascular en la edad adulta (2) por lo que es necesario estudiar el comportamiento de estas variables a efectos de fortalecer el conocimiento local de esta problemática en la población infantil y sugerir los correctivos pertinentes para la implementación de estrategias contextualizadas.

\section{MÉTODOS}

Se realizó un estudio cuantitativo descriptivo de corte transversal con el objetivo de determinar la presencia de factores de riesgo cardiovascular en 173 niños entre 7 y 14 años, con una edad promedio de 9,9 años (IC95 \% 9,6-10,3) que asistían a un colegio de estrato medio-bajo de Cartagena de Indias, durante el año 2009. 
A todos los participantes, en compañía de sus padres o representantes legales, en razón de tratarse de menores de edad, se les explicó los objetivos, riesgos y beneficios del estudio y se les solicitó firma de una cláusula de consentimiento informado elaborada de acuerdo con la normatividad vigente para estudios en humanos, resolución 08430/1993 del Ministerio de Salud de Colombia.

Todos los protocolos del estudio fueron sometidos a evaluación y aprobación por parte del Comité Ético de la Universidad de San Buenaventura Seccional Cartagena.

Se incluyó la totalidad de los niños de la institución educativa que cumplían con la edad estipulada como criterio de inclusión (7-14 años). El grupo estudiado quedó conformado por 86 (49\%) niños y 87 (51\%) niñas, con edades promedios de 9,9 y 10,0 años, respectivamente, sin diferencia significativa (niñas IC95 \%: 9,4-10,4; niños IC95\%: 9,6-10,4; $\mathrm{p}=0,615$ ).

Las muestras de sangre fueron tomadas en ayunas por venopunción. Para las determinaciones de glucemia y perfil lipídico se separaron los sueros de manera inmediata y se procesaron en el analizador automatizado HumaStar80 (Human GmbH, Germany), mediante técnicas enzimáticas espectrofotométricas, utilizando controles de calidad internos y externo a fin de validar los resultados obtenidos.

Los valores correspondientes a niveles en suero de triglicéridos, colesterol total, colesterol no asociado a lipoproteínas de alta densidad (Colesterol no HDL), y sus fracciones en lipoproteínas de baja (C-LDL) o alta densidad (C-HDL), se categorizaron en valores deseables, de alarma o alterados, de acuerdo con las recomendaciones de los expertos del National Cholesterol Education Program (NCEP) (3).

Las alteraciones de peso corporal fueron establecidas utilizando las tablas de Índice de Masa Corporal (IMC) para edad y sexo avaladas por los Centros para el Control y Prevención de Enfermedades (CDC, por sus siglas en inglés Centers for Disease Control and Prevention) el cual define obesidad cuando el IMC supera el percentil 95, sobrepeso cuando se halla entre los percentiles 85 y 95 y peso bajo cuando sea inferior al percentil cinco (4). 


\section{RESULTADOS}

Cuando se trata de mejorar los perfiles de riesgo cardiovascular, se entiende que tal acción solo resultará efectiva para los factores de riesgo susceptibles de ser modificados. Sin embargo, su estudio es obligado, si se tiene en cuenta que la contribución de la historia familiar aporta un riesgo independiente incluso del conferido por los otros factores tradicionalmente aceptados (5).

Por lo anterior, se indagó la presencia de antecedentes de enfermedad cardiovascular en familiares de primer y segundo grado de consanguinidad, como factor no modificable, la cual se presumía que debía estar elevada si se toma en consideración el comportamiento de las causas de morbimortalidad del departamento en lo que se refiere a este tipo de enfermedades. Solamente $43(24,9 \%)$ de los niños entrevistados expresaron no presentar antecedentes de este tipo. El resto presentó uno o más antecedentes, siendo los más frecuentes hipertensión $(105 / 173 ; 60,7 \%$; IC $95 \%$ 53,4-68,0), hipercolesterolemia $(83 / 173 ; 48,0 \%$; IC $95 \% 40,6-55,4)$ y diabetes mellitus $(66 / 173 ; 38,2 \%$; IC $95 \% 31,0-45,4)$.

En adición a la historia familiar para eventos cardiovasculares, se estudió la presencia de factores de riesgo personales, bioquímicos y conductuales. En tal sentido, las concentraciones promedio de las variables bioquímicas como concentraciones de lípidos y glucemia y antropométricas, como IMC, se ubicaron dentro de los límites deseables (Tabla 1), sin embargo, al clasificar las concentraciones del perfil lipídico que presentó cada niño estudiado, se evidencia que un número considerable de ellos se ubican entre valores de niveles de alarma y alterados, de acuerdo con los límites recomendados por el NCEP (Figura 1). Todos los niños presentaron niveles de glucemia dentro de valores de referencia.

Tabla 1. Estadística descriptiva del comportamiento de las variables antropométricas y bioquímicas de la población

\begin{tabular}{lccc}
\hline \multicolumn{1}{c}{ Variable } & Media & Desviación estándar & IC95 \% \\
\hline IMC $\left(\mathrm{kg} / \mathrm{m}^{2}\right)$ & 15,5 & 2,4 & $15,1-15,8$ \\
Colesterol total $\mathrm{mg} / \mathrm{dL})$ & 174,7 & 29,7 & $170,2-179,1$ \\
Colesterol HDL $(\mathrm{mg} / \mathrm{dL})$ & 49,7 & 6,7 & $48,7-50,7$ \\
Colesterol no HDL $(\mathrm{mg} / \mathrm{dL})$ & 124,4 & 24,3 & $120,7-128,0$ \\
Colesterol LDL $(\mathrm{mg} / \mathrm{dL})$ & 107,3 & 23,6 & $103,8-110,9$ \\
Triglicéridos $(\mathrm{mg} / \mathrm{dL})$ & 87,9 & 33 & $83,0-92,9$ \\
Glucemia $(\mathrm{mg} / \mathrm{dL})$ & 87,4 & 9,7 & $86,0-88,9$ \\
\hline
\end{tabular}


La comparación de los niveles en suero de lípidos, glucemia e IMC según sexo solo arrojó diferencia significativa para niveles de triglicéridos, siendo mayor en niñas $(94,1 \mathrm{mg} / \mathrm{dL}$; IC95 \% 93,0-95,2) que en varones $(81,7 \mathrm{mg} /$ $\mathrm{dL} ;$ IC95 \% 80,6-82,8) (p=0,005).

Figura 1. Distribución porcentual de acuerdo con el nivel de las variables bioquímicas estudiadas

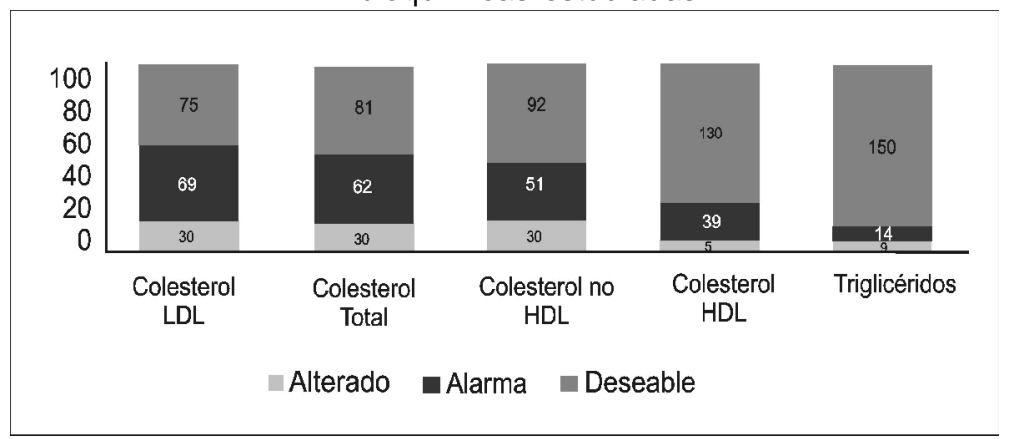

El IMC en niños y adolescentes ha demostrado ser uno de los mejores indicadores de alteraciones del peso corporal (6). Aplicando las curvas para edad y sexo del CDC para este indicador, del total de 173 niños estudiados, $50(28,9 \%$; IC95 \% 22,2-35,7) presentaron bajo peso, $116(67,1 \%$; IC95 \% $60,1-74,1)$ peso saludable, $4(2,3 \%$; IC95 \% $0,1-4,5)$ sobrepeso y $3(1,7 \%$; IC95 \% 0,0-3,6)) obesidad, tal como se muestra en la Figura 2, donde puede observarse adicionalmente que, aunque la mayor parte de los niños se distribuyen entre IMC que corresponden a peso bajo y saludable, en todos los casos la presencia de alteraciones de lípidos en suero fue elevada.

En el grupo estudiado, la presencia de escaso tiempo dedicado al ejercicio físico (menor de 2 horas de ejercicio físico vigoroso por semana) está presente en el 69,9\% de los niños incluidos en el estudio (IC $95 \%$ 63,1-76,5\%).

Sin embargo, al comparar entre sexos, este comportamiento se observa con más frecuencia en las niñas $(83,8 \%$; IC $95 \%$ 73,5-94,5 \%) que en los niños $(44,2 \%$; IC95 \% 45,4-66,6), con diferencia estadísticamente significativa $(\mathrm{p}=0,0001)$. 
Observando el tipo de actividad física preferida para los momentos de esparcimiento, los hallazgos son similares. El 51,4\% (89/173) (IC $95 \%$ 44,0$58,9)$ de la población escogió como sus favoritas actividades sedentarias, tales como juegos de computador, ver televisión o leer.

Figura 2. Distribución porcentual de la presencia de alteraciones en alguno de los componentes del perfil lipídico, de acuerdo con las categorías de peso corporal definidas por IMC

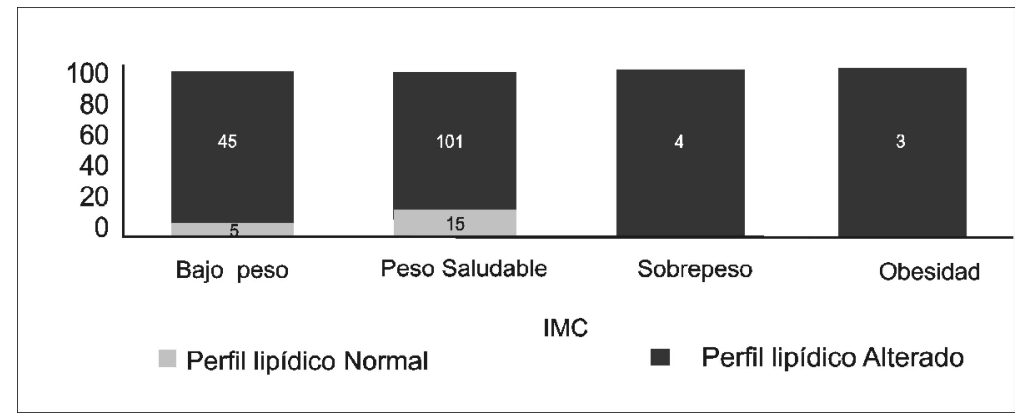

Al comparar por sexo, el 65,5 \% (57/87) (IC $95 \%$ 55,0-76,0) de las niñas expresaron preferencia por actividades recreativas poco activas mientras que esa respuesta solo fue evidenciada en el 37,2 \% (32/86) (IC $95 \%: 26,6-$ $47,8)$ de los niños, siendo esta diferencia estadísticamente significativa $(\mathrm{p}=0,0001)$.

Considerando el efecto sinérgico que se ha demostrado en los factores de riesgo antes mencionados (antecedentes familiares, sedentarismo, dislipidemias y sobrepeso u obesidad), se estudió en cada niño la presencia simultánea de los mismos. Los resultados mostraron que el 1,2\% (2/173) de los niños estaba exento de ellos, el 19, $0 \%$ (33/173) presentaba un único factor de riesgo, el 43,9\% (76/173) y el 35,3\% (61/173) presentaron al tiempo dos y tres factores, respectivamente. El 0,6\% (1/173) de los niños presentaron los cuatro factores de manera simultánea.

\section{DISCUSIÓN}

El carácter multifactorial de la enfermedad cardiovascular obliga a que se incluyan entre sus factores de riesgo tanto aquellos de naturaleza biológica como los psicosociales. La elevada presencia de antecedentes familiares encontrada en el 
presente estudio indica, por un lado, una importante predisposición genética y, por el otro, una elevada probabilidad de desarrollar y sostener en el tiempo estilos de vida, nocivos para la salud cardiovascular, aprendidos o fácilmente influenciables por el entorno familiar más o menos inmediato (7).

De acuerdo con los valores de IMC hallados en este estudio, la mayor parte de los niños cursan con peso normal o bajo, mientras un $4 \%(7 / 173)$ cursa con IMC indicativo de sobrepeso u obesidad, coincidente con lo hallado en una población similar en Venezuela, durante el año 2003 (8), situación muy distinta cuando se compara con niños europeos (9), tal como era esperable en virtud de tratarse de contextos sociales y económicos marcadamente diferentes.

Por otra parte, aunque el bajo peso no represente riesgo desde la perspectiva de la salud cardiovascular, es importante destacar este hallazgo en 50 de los 173 niños estudiados, toda vez que pone en evidencia una situación que debe ser intervenida a efectos de asegurar un normal y correcto desarrollo de las capacidades físicas, mentales y biológicas en esta importante etapa de los infantes.

En contraste, el impacto de los porcentajes obtenidos de obesidad y sobrepeso deberá considerarse teniendo en cuenta la edad del grupo, y la evidencia de estudios de seguimiento en niños que han demostrado que el incremento de IMC en esta etapa es predictor de obesidad y riesgo cardiovascular en la edad adulta (10), sin olvidar la tendencia mundial hacia el incremento de la prevalencia de obesidad y sobrepeso que se calcula se ha triplicado en las últimas dos décadas (11-13).

Adicionalmente, la frecuencia de alteraciones en el perfil lipídico fue elevada, incluso en aquellos niños con peso corporal normal o bajo. Si bien es conocida la relación entre presencia de obesidad infantil y dislipidemias (14), el grupo estudiado, a pesar de tener baja frecuencia de obesidad y sobrepeso, mostró alteraciones de los perfiles lipídicos, situación que hubiera pasado inadvertida si la solicitud de tales perfiles se condicionara a la presencia de estados de sobrepeso u obesidad.

Los resultados mostraron alteración especialmente de colesterol total y su fracción LDL, lo cual coincide con lo reportado por otros autores $(15,16)$ y alerta sobre la urgencia de adelantar intervenciones en este sentido. 
El colesterol no HDL también se encontró elevado en un porcentaje importante de los niños estudiados. Estudios poblacionales de seguimiento de este factor, como el Bogalusa Heart Study, han demostrado que logra predecir de manera efectiva el desarrollo de dislipidemias, obesidad y riesgo cardiovascular en los adultos, inclusive de mejor manera que el tradicional colesterol de lipoproteínas de baja densidad (17).

Entre las principales herramientas que se han comprobado eficaces para mejorar la salud cardiovascular se encuentra el ejercicio físico regular. Sus efectos sinérgicos favorecen cambios conductuales y mejoran los perfiles bioquímicos $(18,19)$. La importancia del ejercicio físico como comportamiento deseable en este momento específico del desarrollo de los niños radica en que durante este período se consolidan hábitos que perdurarán toda la vida.

No obstante, el incremento de nuevas tecnologías con alternativas lúdicas sedentarias y la reducción de espacios recreacionales seguros, ha generado una notable disminución en la actividad física de niños y adolescentes, especialmente en las zonas urbanas (20), siendo este comportamiento más notorio en niñas que en niños, lo que coincide con lo encontrado en este estudio $(21,22)$.

Las enfermedades cardiovasculares ocupan los primeros lugares de causas de mortalidad general en la población adulta del distrito de Cartagena de Indias, Colombia, tal como se observa en los reportes oficiales de los últimos años (23). Sin embargo, su condición de enfermedad crónica de inicio temprano y fuertemente ligada al sostenimiento de factores de riesgo muchos de ellos modificables, hace eficaz el desarrollo de estrategias de prevención primaria, las cuales deberán soportarse en el conocimiento del estado actual de riesgo cardiovascular en que se hallan las poblaciones jóvenes, y en experiencias exitosas culturalmente adaptadas, que permitan identificar tempranamente la presencia de riesgo cardiovascular y fomentar la adhesión a estilos de vida saludables (25-29).

Aunque la evidencia actual presenta debilidad a la hora de poder establecer como recomendación la evaluación de lípidos en la totalidad de la población infantil (30), ésta debería empezar a ser considerada si se desea evitar que las curvas tendenciales de la enfermedad cardiovascular sigan en ascenso y esta enfermedad continúe siendo la responsable del mayor número de muertes en nuestra región. 
En coincidencia con lo expresado por otros autores, este estudio demostró la presencia de factores de riesgo cardiovascular en niños y adolescentes, inclusive en ausencia de obesidad, lo que indica que los individuos metabólicamente obesos pero con peso normal pueden ser identificados desde edades tempranas (31) e intervenidos, evitando la progresión de la enfermedad.

En resumen, los resultados hallados en esta población infantil demuestran la necesidad de continuar con el estudio y seguimiento de las condiciones que subyacen y soportan los perfiles de morbimortalidad de las regiones, indagando e interviniendo oportunamente marcadores cardiovasculares tradicionales y no tradicionales (16) a fin de provocar un impacto positivo en la calidad de vida presente y futura de estas poblaciones.

En tal sentido, las universidades en asocio con estamentos públicos y privados, están llamados a dar respuesta a esta problemática a fin de superar la mecánica histórica de programas bienintencionados pero que carecen en muchas oportunidades de continuidad y de conocimiento veraz de la realidad que intentan impactar. A partir de trabajos como el presente se tienen presupuestadas fases posteriores que complementen el abordaje desde miradas mixtas que incluyan el componente cualitativo y ayuden a comprender los procesos que, desde la infancia, están llevando a nuestras poblaciones hacia una adultez con mala alimentación, sedentarismo y obesidad, sustrato perfecto para el incremento de las prevalencias de enfermedad y muerte por causas cardiovasculares

Agradecimientos: A las directivas de la Universidad de San Buenaventura, Seccional Cartagena, y a la población de niños y padres que prestaron su colaboración e hicieron posible este trabajo.

\section{REFERENCIAS}

1. Vega de Céniga M. Participación de la lipoproteína de baja densidad oxidada en el desarrollo de la placa ateroesclerótica. Angiología 2006; 58(1):51-56.

2. Magnussen C, Venn A, Thomson R, Juonala M, Srinivasan S, Viikari J, et al The Association of Pediatric Low- and High-Density Lipoprotein Cholesterol Dyslipidemia Classifications and Change in Dyslipidemia Status With Carotid Intima-Media Thickness in Adulthood: Evidence From the Cardiovascular Risk in Young Finns Study, the Bogalusa Heart Study, and the CDAH (Childhood Determinants of Adult Health) Study J. Am. Coll. Cardiol. 2009; 53:860-869.

3. National Cholesterol Education Program (NCEP): Highlights of the Report of the Expert Panel on Blood Cholesterol Levels in Children and Adolescents. Pediatrics 1992; 89:495501. 
4. Centers for Disease Control and Prevention [Internet]. About BMI for Children and Teens. CDC BMI-for-age growth charts for girls and boys. Disponible en: http:// www.cdc.gov/growthcharts Consultado: Agosto de 2009.

5. Valdez R, Greenlund KJ, Khoury MJ, Yoon PW . Is Family History a Useful Tool for Detecting Children at Risk for Diabetes and Cardiovascular Diseases? A Public Health Perspective. Pediatrics 2007; 120:S78-S86.

6. Mei Z, Grummer LM, Pietrobelli A, Goulding A, Goran MI, Dietz WH. Validity of body mass index compared with other body-composition screening indexes for the assessment of body fatness in children and adolescents. Am J Clin Nutr 2002; 75:978-85.

7. Sánchez M, Moreno GA, Marín ME, García LH. Factores de Riesgo Cardiovascular en Poblaciones Jóvenes Cardiovascular. Rev. salud pública (Bogotá). 2009; 11(1):110122.

8. Solano L, Velásquez E, Naddaf G, Páez M. Patrón de lípidos en preescolares de bajos recursos socioeconómicos (Valencia, Venezuela) ACV 2003; 54:254-262.

9. Muros JJ, Som A, Zabala M, Oliveras MJ, López SH. Evaluación del estado nutricional en niños y jóvenes escolarizados en Granada. Nutr clín diet hosp 2009; 29(1):26-32.

10. Freedman DS, Khan LK, Serdula MK, Dietz WH, Srinivasan SR, Berenson GS. The Relation of Childhood BMI to Adult Adiposity: The Bogalusa Heart Study. Pediatrics. 2005; 115:22-27.

11. US Preventive Services Task Force. Screening and Interventions for Overweight in Children and Adolescents: Recommendation Statement. Pediatrics. 2005; 116:205-209.

12. Spiotta RT, Luma GB. Evaluating Obesity and Cardiovascular Risk Factors in Children and Adolescents. Am Fam Physician. 2008; 78(9):1052-1058.

13. Copeland KC, Becker D, Gottschalk M, Hale D. Type 2 Diabetes in Children and Adolescents: Risk Factors, Diagnosis, and Treatment. Clin Diabetes. 2005; 23(4):181-185.

14. Gotthelf SJ, Jubany LL. Antropometría y lípidos séricos en niños y adolescentes obesos de la ciudad de Salta, 2006. Arch Argent Pediatr 2007; 105(5):411-417.

15. Steinberger J, Daniels SR. Obesity, Insulin Resistance, Diabetes, and Cardiovascular Risk in Children An American Heart Association Scientific Statement From the Atherosclerosis, Hypertension, and Obesity in the Young Committee (Council on Cardiovascular Disease in the Young) and the Diabetes Committee (Council on Nutrition, Physical Activity, and Metabolism). Circulation. 2003; 107:1448-1453.

16. Villarreal E, Forero Y, Poveda E, Baracaldo C, López E. Marcadores de riesgo cardiovascular en escolares de cinco departamentos de la región oriental en Colombia. Biomédica. 2008; 28:38-49.

17. Srinivasan SR, Frontini MG, Xu J, Berenson GS. Utility of Childhood Non-High-Density Lipoprotein Cholesterol Levels in Predicting Adult Dyslipidemia and Other Cardiovascular Risks: The Bogalusa Heart Study. Pediatrics. 2006; 118:201-206.

18. Perichart O, Balas M, Ortiz V, Morán JA, Guerrero JL, Vadillo F. Programa para mejorar marcadores de riesgo cardiovascular en escolares mexicanos. Sal Pub Mex. 2008; $50(3): 218-226$

19. Ho TF. Cardiovascular Risks Associated With Obesity in Children and Adolescents, Ann Acad Med Singapore. 2009; 38:48-56.

20. Cornejo J, Llanas JD, Alcázar C. Acciones, programas, proyectos y políticas para disminuir el sedentarismo y promover el ejercicio en los niños. Bol Med Hosp Infant Mex. 2008; 65(6):616-25.

21. McDermott RJ, Nickelson J, Baldwin JA, Bryant CA, Alfonso M, Phillips LM, et al. Alianza entre la comunidad, el distrito escolar y la Universidad para evaluar la actividad física en los preadolescentes. Prev Chronic Dis. 2009; 6(1). Disponible en: http:// www.cdc.gov/pcd/issues/2009/jan/07_0243_es.htm. Consultado Mayo 2010.

22. Reed KE, Warburton DER, McKay HA. Determining cardiovascular disease risk in elementary school children: developing a healthy heart score. J Sports Sci. 2007; 6:142148. 
23. Departamento Administrativo Distrital de Salud (DADIS) [Internet]. Perfil epidemiológico de la ciudad de Cartagena de Indias, Bolívar Año 2008. Disponible en: http:// www.dadiscartagena.gov.co/web/images/docs/perfil_epidemiologico_2008.pdf Consultado Mayo 2010.

24. Williams CL, Strobino BA, Bollella M, Brotanek J. Cardiovascular Risk Reduction in Preschool Children: The "Healthy Start" Project. J Am Col Nutr 2004; 23(2):117-123.

25. Daniels SR, Greer FR and and the Committee on Nutrition. Lipid Screening and Cardiovascular Health in Childhood. Pediatrics. 2008; 122(1) 198-208.

26. US Preventive Services Task Force Screening for Obesity in Children and Adolescents: US Preventive Services Task Force Recommendation Statement. Pediatrics. 2010; 125(2):361-367.

27. Committee on Nutrition Prevention of Pediatric Overweight and Obesity. Pediatrics. 2003; 112(2):424-430.

28. Gidding SS, Dennison BA, Birch LL, Daniels SR, Gilman MW, MD, Lichtenstein AH, et al. Dietary Recommendations for Children and Adolescents: A Guide for practitioners. Pediatrics. 2006; 117(2):544-559.

29. McWilliams C, Ball SC, Benjamin SE, Hales D, Vaughn A, Ward DS. Best-Practice Guidelines for Physical Activity at Child Care. Pediatrics. 2009;124;1650-1659.

30. U.S. Preventive Services Task Force. Screening for Lipid Disorders in Children. US Preventive Services;Task Force recommendation statement. Pediatrics. 2007; 120(1):e215-9.

31. Salazar VB, Rodríguez MM, Guerrero RF Factores bioquímicos asociados a riesgo cardiovascular en niños y adolescentes. Rev Med IMSS. 2005; 43 (4): 299-303. 